\title{
ACIDENTE COM MATERIAL BIOLÓGICO EM SERVIDORES DA SAÚDE NA BAHIA
}

\author{
Ticiana Assemany Cruz*, Cleidiana Oliveira Safira Andrade*, Augusto Cesar Costa Cardoso** \\ Autora para correspondência: Ticiana Assemany Cruz - tassemany@hotmail.com \\ * Graduada em enfermagem pela Escola Bahiana de Medicina e Saúde Pública \\ ** Doutor e pós-doutor em Saúde Pública pelo Instituto de Saúde Coletiva/Universidade Federal da Bahia. Professor da \\ UNEB e da Escola Bahiana de Medicina e Saúde Pública
}

\begin{abstract}
Resumo
Os objetivos da pesquisa são descrever o perfil das ocorrências de acidentes de trabalho com material biológico em servidores da saúde no Estado da Bahia, além de identificar o perfil dos profissionais acidentados e delinear o tipo de exposição nos acidentes com material biológico. Trata-se de um estudo epidemiológico, retrospectivo, descritivo com abordagem quantitativa. A coleta de dados foi realizada no banco de dados do SINAN. Os dados foram analisados no Excel (2007). No período de cinco anos (2011 a 2015) foi notificado um total de 10.863 casos de acidente de trabalho com material biológico, sendo o ano de 2014 os profissionais estavam com maior risco, pois representou a taxa de $16,6 \%$ (2.506). O perfil é predominantemente com nível técnico (73\%), sexo feminino (78\%), a faixa etária de 30 a 39 anos (36,3\%). O material biológico com maior transmissão é o sangue (87\%) e a sorologia positiva para HIV apresentam 57 casos. Conclui-se que o conhecimento de dados epidemiológicos na instituição pode contribuir na compreensão de cenários de riscos nos quais os trabalhadores estão expostos e nesse contexto intervir com políticas e programas que visam estratégias de prevenção.
\end{abstract}

Palavras-chave: Saúde do trabalhador; Material biológico; Acidentes de trabalho.

\section{ACCIDENTS INVOLVING BIOLOGICAL MATERIAL AMONG HEALTH SERVERS IN BAHIA}

\begin{abstract}
This study aims to describe the profile of occupational accident with exposure to biological material among health servers, in Bahia, in addition to identifying the profile of the worker exposed to biological material and outline the type of exposure in accidents with biological materials. This is a retrospective and descriptive epidemiological study with a quantitative approach. Data were collected from SINAN database. Data were
\end{abstract}

- Artigo submetido para avaliação em 12/06/2016 e aceito para publicação em 14/06/2016 • 
analyzed in Excel (2007). A total of 10,863 cases of occupational accidents with biological material were notified within five years (2011-2015). In 2014, professionals were more exposed to the risk of suffering an accident at work with biological material, it represents a rate of $16.6 \%(2,506)$. The profile is predominantly composed of female $(78 \%)$ technicians $(73 \%)$, from 30 to 39 years old $(36.3 \%)$. Blood is the biological material with higher contamination rate (87\%). Positive serology for HIV was found in 57 cases. It was concluded that knowledge of epidemiological data in the institution can contribute to the understanding of risk scenarios in which workers are exposed and thus create prevention policies and programs.

Keywords: Occupational Health; Biocompatible Materials; Accidents Occupational.

\section{INTRODUÇÃO}

O trabalho que tornou possível a humanização do homem tem produzido desafios para a área da saúde. O crescimento industrial, os avanços tecnológicos são marcados por intensas e rápidas transformações, que apesar das facilidades e dos benefícios expõe o profissional a riscos ambientais e organizacionais. $O$ processo produtivo no trabalho podem ocasionar doenças ocupacionais ou acidentes de trabalho, comprometendo à saúde do trabalhador. (1)

$O$ acidente de trabalho é conceituado pela Lei $n^{\circ}$ 8.213 de 24 de julho de $1991^{(2)}$ como "aquele que ocorre pelo exercício do trabalho, a serviço da empresa, ou ainda pelos segurados especiais, provocando lesão corporal ou perturbação funcional, que cause a morte ou redução da capacidade do trabaIho, permanente ou temporário". Os acidentes de trabalho resultam em danos físicos e/ou psíquicos levando prejuízos nas relações sociais e familiares.

A Organização Internacional do Trabalho (OIT) descreve o ambiente de trabalho decente como aquele que é produtivo e de qualidade, em condições de liberdade, equidade, segurança e dignidade humanas. (3) E para atingir esse ideal, órgãos nacionais e internacionais buscam desenvolver programas e políticas com medidas preventivas que evitem acidentes de trabalho. ${ }^{(4)}$
O acidente no exercício da profissão é considerado um problema de saúde publica em todo o mundo, apesar das subnotificações, as estatísticas trazem números relevantes, considerado uma pandemia oculta. Dados divulgados pela Organização das Nações Unidas (ONU) em 2013(5) demonstraram que 321 mil pessoas morrem a cada ano, estima-se que a cada 15 segundos 153 trabalhadores sofrem um dano. O Brasil está classificado como o quarto colocado no ranking mundial quanto aos acidentes fatais de trabalho.

Trabalhadores da área da saúde estão mais expostos a adquirir infecções do que o resto da população e, em geral, há associação com as atividades desempenhadas. Observa-se que na sua maioria por contato direto com pacientes debilitados e acometidos por diversos patógenos potencialmente transmissíveis, como a tuberculose, pneumonias, vírus da hepatite $B(\mathrm{HBV})$, o hepatite C (HVC), o HIV, entre outros. ${ }^{(6)}$

As vacinas são consideradas como barreiras imunológicas, portanto devem estar sempre atualizado o cartão dos profissionais de saúde, como também o uso de equipamentos de proteção individual (EPI) e coletiva (EPC) são essenciais para minimizar a exposição. ${ }^{(6)}$ 
As normas de biossegurança são fundamentais no processo de trabalho garantindo a estes, padrões mínimos de segurança, entretanto profissionais de diferentes categorias ainda demonstram grande resistência em adotar as medidas preventivas recomendadas, ficando vulnerável aos acidentes. ${ }^{(7)}$

A promoção em educação em saúde, o autocuidado profissional, e a equipe da medicina ocupacional devem ir a campo estudar os ambientes de trabalho para identificar os possíveis riscos e as medidas de prevenção, são propostas que devem permear a cultura organizacional. ${ }^{(6)}$

O interesse pela temática acidente de trabalho com exposição a material biológico envolvendo profissionais da saúde percorre do crescente número de notificações, falta de políticas e programas que tenham a perspectiva de controlar ou prevenir esses agravos.

O objetivo geral é descrever o perfil das ocorrências de acidentes de trabalho com exposição a material biológico, na Bahia no período compreendido entre 2011 a 2015 e os objetivos específicos são: identificar o perfil dos profissionais acidentados por material biológico e delinear o tipo de exposição nos acidentes com material biológico.

\section{METODOLOGIA}

Trata-se de um estudo epidemiológico retrospectivo, descritivo, com abordagem quantitativa, realizado por meio de busca em base de dados secundário no Estado da Bahia, Brasil.

A operacionalização do processo ocorreu através de consultas no sistema DATASUS (TabNet) na área de saúde do trabalhador, que consta o banco de dados do Sistema Nacional de Agravos Notificáveis (SINAN) selecionado apenas as notificações no período de 2011 à 2015.

As variáveis de estudos são: sexo (feminino e masculino), faixa etária (18-59 anos), ocupação, selecionados apenas trabalhadores na área da saúde (graduados, nível técnicos na área da ciência e bioquímica, trabalhadores do serviço de saúde), materiais orgânicos com números relevantes (sangue, liquido pleural, fluido com sangue, soro/plas ma e outros) e os agentes biológicos (anti-HIV, anti-HCV, anti-HBV e HbsAg). Dados com informações: inconclusivos, não realizado ou ignorado, foram excluídos.

Realizou-se analise por meio da exportação de dados do DATASUS (TabNet) para o Excel pacote Office (2007) que possibilitou o processamento dos dados, cálculos dos indicadores de proporção e taxas.

\section{RESULTADOS E DISCUSSÃO}

O contexto político e econômico brasileiro, sobretudo no âmbito dos Serviços Públicos, nas condições para reduzir os gastos, optem pela quantidade mínima de recursos humanos ou pela flexibilização das relações de trabalho, fatores esses que interferem no processo de trabalho e consequentemente na saúde dos trabalhadores. ${ }^{(8)}$

$\mathrm{Na}$ literatura o conceito trabalho precário pode variar como sendo uma situação de déficit ou ausência de seus direitos e proteção social nas relações de trabalho, instabilidade de vinculo, ou ainda associada às condições de trabalho em determinados setores da economia que geram vulnerabilidade aos profissionais quando inseridos nessas condições. ${ }^{(8)}$

No setor público brasileiro, especialmente o da saúde, a existência do trabalho precário causa grande consequências aos profissionais, que por receio de ficarem desempregados acabam se sujeitando à condição de ausência de proteção social, desempenhando sua atividade com poucos recursos materiais, sobrecarga de trabalho e principalmente fragilizando o processo de cuidar do outro. ${ }^{(8)}$

Os serviços de saúde precisam ser espaços salubres, que a cultura organizacional ofereça à garantia da atenção integral a saúde, com acesso universal, igualitário a toda população, como está preconizado na Lei $8080 / 90{ }^{(9)}$ 
Tabela 1 - Taxa de incidência (em 100.000) de investigações por acidente de trabalho com material biológico. Bahia, 2011-2015

\begin{tabular}{ccc}
\hline ANO DA NOTIFICAÇÃO & INVESTIGAÇÕES & TAXA \\
\hline 2011 & 1.808 & 12,8 \\
2012 & 1.837 & 13,0 \\
2013 & 2.220 & 14,8 \\
2014 & 2.506 & 16,6 \\
2015 & 2.492 & 16,4 \\
\hline Total & 10.863 & - \\
\hline
\end{tabular}

*Fonte: SINAN NET, Bahia, Brasil 2016.

As informações geradas, segundo o indicador de taxa, possibilita estimar o risco de um trabalhador sofrer acidente com material biológico no estado da Bahia.

No período de cincos anos é perceptível o aumento de notificações nas investigações dos acidentes, em 2011 foram 1.808 (12,8\%), quando comparado a 2013 e 2014 observa-se um aumento nas taxas de $14,8 \%$ para $16,6 \%$, respectivamente.
O ano de 2014 os profissionais estavam mais expostos ao risco de sofrer um acidente de trabalho com material biológico, representam os maiores casos registrados (2.506) no período em estudo.

Compreendendo que a taxa é um indicador de segurança no trabalho e da eficácia das medidas preventivas adotadas pelas empresas, as estratégias adotadas não estão sendo efetivas, tendo em vista o crescimento das notificações.

Tabela 2 - Distribuição dos acidentes de trabalho com material biológico segundo ano de notificação e a caracterização do perfil profissional. Bahia, 2011-2015

\begin{tabular}{|c|c|c|c|c|c|c|c|c|c|c|c|c|}
\hline \multirow{2}{*}{$\frac{\text { ANO }}{\text { VARIÁVEIS }}$} & \multicolumn{2}{|c|}{2011} & \multicolumn{2}{|c|}{2012} & \multicolumn{2}{|c|}{2013} & \multicolumn{2}{|c|}{2014} & \multicolumn{2}{|c|}{2015} & \multicolumn{2}{|c|}{ TOTAL } \\
\hline & No & $\%$ & No & $\%$ & No & $\%$ & No & $\%$ & No & $\%$ & No & $\%$ \\
\hline \multicolumn{13}{|l|}{ Ocupação } \\
\hline Médicos e enfermeiros & 307 & 16 & 353 & 18 & 406 & 21 & 449 & 23 & 409 & 21 & 1924 & 10 \\
\hline Técnicos de enfermagem e afins & 939 & 17 & 983 & 18 & 1146 & 21 & 1259 & 23 & 1095 & 20 & 5422 & 10 \\
\hline Técnicos de bioquímica e afins & 3 & 13 & 4 & 17 & 4 & 17 & 6 & 26 & 6 & 26 & 23 & 10 \\
\hline $\begin{array}{l}\text { Trabalhadores dos serviços de } \\
\text { saúde }\end{array}$ & 30 & 21 & 23 & 16 & 35 & 25 & 22 & 16 & 30 & 21 & 140 & 10 \\
\hline
\end{tabular}

Sexo

$\begin{array}{lcccccccccccc}\text { Masculino } & 381 & 17 & 370 & 16 & 457 & 20 & 556 & 24 & 542 & 24 & \mathbf{2 3 0 6} & \mathbf{1 0 0} \\ \text { Feminino } & 1389 & 17 & 1416 & 17 & 1704 & 21 & 1862 & 23 & 1844 & 22 & \mathbf{8 2 1 5} & \mathbf{1 0 0}\end{array}$

Faixa etária

\begin{tabular}{|c|c|c|c|c|c|c|c|c|c|c|c|}
\hline 20 a 29 anos & 678 & 18 & 646 & 17 & 822 & 22 & 875 & 23 & 762 & 20 & 3783 \\
\hline 30 a 39 anos & 617 & 16 & 627 & 17 & 762 & 20 & 859 & 23 & 925 & 24 & 3790 \\
\hline 40 a 49 anos & 311 & 16 & 345 & 18 & 393 & 20 & 454 & 23 & 449 & 23 & 1952 \\
\hline 50 a 59 anos & 155 & 17 & 151 & 17 & 170 & 19 & 213 & 24 & 213 & 24 & 902 \\
\hline
\end{tabular}

*Fonte SINAN NET, Bahia, Brasil 2016. 
A variável ocupação profissional através dos dados é possível compreender que os trabalhadores que desempenham funções em contato direto com os pacientes e manipulação com material perfurocortante ao longo da jornada de trabalho estão mais expostos ao risco de acidente. Os resultados mais significativos estão relacionados aos acidentes envolvendo os profissionais de nível técnico $6.110(73 \%)$, seguidos por enfermeiros e médicos 2.099 (25\%), os trabalhadores dos serviços de saúde $161(1,9 \%)$ e os técnicos em bioquímica e áreas afins $24(0,3 \%)$.

O estudo realizado no CEREST de Florianópolis apresentam dados semelhantes, profissionais técnicos de enfermagem $(38,26 \%)$ assumem atividades como punção venosa e administração de medicamento endovenosa, os enfermeiros da assistência que promovem trocas de curativos e manuseio de sondas, por exemplo, e os médicos na especialidade de cirurgia principalmente são os segundos mais expostos $(13,05 \%)$, e, outros trabalhadores na saúde $(11,30 \%)$, por exemplo, os agentes de limpeza que coletam o lixo que muitas vezes encontra-se com descartes inadequados de perfurocortante e material contaminado. ${ }^{(10)}$

Cabe destacar que trabalhadores de diferentes categorias profissionais, enfermeiro, médico, técnico de enfermagem, bioquímica e laboratório e todos os trabalhadores da saúde como os agentes de higienização, são potenciais vitimas de acidentes com exposição a material biológico, logo todo profissional que presta serviço de saúde merece atenção quanto ao planejamento estratégico de biossegurança. ${ }^{(11)}$

Quanto ao sexo, os profissionais do sexo feminino sofreram mais acidentes, chegando a 8.215 casos notificados (78\%) e durante todos os cinco anos representados, as mesmas têm a prevalência em até quatro vezes maior quando comparadas ao sexo masculino, com 2.306 notificações $(22 \%)$.

Compreende-se que as mulheres é o maior grupo prestador de assistência a saúde que estão expostas aos riscos biológicos. Esse dado é reflexo do grande contingente composto pela equipe de enfermagem, a qual historicamente é exercida predominantemente pelo sexo feminino. ${ }^{(4)}$

A incidência de trabalhadoras a exposição a material biológico coincide na literatura nacional e internacional, ${ }^{(4)}$ inclusive pela classe desempenhar o papel de cuidar vinte e quatro horas por dia, responsável por cerca de $60 \%$ da execução dos procedimentos de saúde, estando constantemente em contato com o paciente e pela atividade de trabalho necessitar de manuseio com material biológico, são os principais fatores de risco para ocorrência de acidente ocupacional. ${ }^{(6)}$

De acordo a faixa etária os trabalhadores jovens são mais acometidos, apresentando a faixa etária 30 a 39 anos com 3.790 registros (36,3\%), e 20 a 29 anos com $3.783(36,3 \%)$, diferença de sete casos, representando assim o mesmo valor percentual. Na terceira colocação segue as trabalhadoras de 40 a 49 anos com $1.952(18,7 \%)$ registros e na quarta o grupo de 50 a 59 anos com $902(8,7 \%)$ casos.

O resultado apresentando na seguinte tabela esta em concordância com estudo realizado no Hospital das Clinicas da Faculdade de Medicina em São Paulo que encontrou o resultado de $34,4 \%$ predominantemente com profissionais de 30 à 39 anos. ${ }^{(4)}$

A ocorrência dos acidentes com profissionais nesta faixa etária pode ser relacionada à experiência e à prática já adquirida, que leva as trabalhadoras a desenvolver a postura de autoconfiança, a resistência ao uso de Equipamento de Proteção Individual (EPI), além dos fatores externos do ambiente de trabalho que corroboram para o evento, por exemplo, o processo de trabalho. ${ }^{(12)}$

Porém existe uma discordância dessa variável, em outros estudos encontrados, sendo assim, não há uma definição quanto à faixa etária do profissional que sofre mais acidente de trabalho expostos a material biológico, variando de acordo com o perfil da instituição e a subnotificação.

Estudo realizado no Hospital Universitário da Alemanha identificou a informação sobre subnotificação, que foi menor no primeiro acidente com $35,3 \%$, 
aumentou no segundo para $59,3 \%$ e o terceiro acidente $66,7 \%$ dos casos foram notificados. ${ }^{(4)}$

Na Santa Casa de Pelotas, por exemplo, jovens de 21 a 30 anos representam $53,9 \%$ das trabalha- doras acidentadas com material biológico, é justificado pela pouca experiência profissional, insegurança na realização das técnicas ou pela falta de destreza manual destes sujeitos. ${ }^{(11)}$

Tabela 3 - Distribuição dos acidentes de trabalho com material biológico segundo o material orgânico. Bahia, 2011-2015

\begin{tabular}{cccccccccccccc}
\hline ANO & \multicolumn{2}{c}{2011} & \multicolumn{2}{c}{2012} & \multicolumn{2}{c}{$\mathbf{2 0 1 3}$} & \multicolumn{2}{c}{$\mathbf{2 0 1 4}$} & \multicolumn{2}{c}{$\mathbf{2 0 1 5}$} & \multicolumn{1}{c}{ TOTAL } \\
\hline Mat. ORGÂNICO & No & $\%$ & No & $\%$ & No & $\%$ & No & $\%$ & No & \% & No & $\%$ \\
\hline Sangue & 1360 & 17 & 1368 & 17 & 1733 & 22 & 1847 & 23 & 1643 & 21 & $\mathbf{7 9 5 1}$ & $\mathbf{1 0 0}$ \\
Líquido pleural & 1 & 6 & 2 & 13 & 3 & 19 & 1 & 6 & 9 & 56 & $\mathbf{1 6}$ & $\mathbf{1 0 0}$ \\
Fluído com sangue & 67 & 16 & 74 & 18 & 86 & 21 & 106 & 26 & 80 & 19 & $\mathbf{4 1 3}$ & $\mathbf{1 0 0}$ \\
Soro/plasma & 13 & 23 & 2 & 4 & 14 & 25 & 9 & 16 & 19 & 33 & $\mathbf{5 7}$ & $\mathbf{1 0 0}$ \\
Outros & 109 & 16 & 132 & 19 & 133 & 19 & 165 & 24 & 151 & 22 & $\mathbf{6 9 0}$ & $\mathbf{1 0 0}$ \\
\hline
\end{tabular}

*Fonte SINAN NET, Bahia, Brasil 2016.

Em relação ao material orgânico o sangue apareceu na maior proporção no período de cinco anos com 7.951 notificações (87\%) e é o principal responsável por transmissão nos hospitais. Outros fluidos corpóreos passiveis de transmissão correspondem a 690 (8\%): fluídos com sangue foram responsáveis por 413 (5\%) registros, soro/plasma representa 57 (1\%), por fim o liquido pleural com $16(0 \%)$ casos.

$\mathrm{Na}$ Faculdade de Catanduva, foi realizado um estudo qualitativo, onde os profissionais relatam ca- sos com acidentes em contato com sangue, durante procedimentos rotineiros como punção venosa ou na retirada do escalpe, e o evento ocorre principalmente quando não estão fazendo o uso do EPI. ${ }^{(12)}$

Os ferimentos com agulhas ou com outros materiais perfurocortantes que tiveram exposição a materiais orgânicos potencialmente infectantes são extremamente perigosos pela possibilidade do colaborador se contaminar com mais de 20 patógenos diferentes.

Tabela 4 - Distribuição dos acidentes de trabalho com material biológico segundo o ano de notificação e sorologia. Bahia, 2011-2015

\begin{tabular}{|c|c|c|c|c|c|c|c|c|c|c|c|c|c|}
\hline \multirow{2}{*}{\multicolumn{2}{|c|}{$\frac{\text { ANO }}{\text { SOROLOGIA }}$}} & \multicolumn{2}{|c|}{2011} & \multicolumn{2}{|c|}{2012} & \multicolumn{2}{|c|}{2013} & \multicolumn{2}{|c|}{2014} & \multicolumn{2}{|c|}{2015} & \multicolumn{2}{|c|}{ TOTAL } \\
\hline & & No & $\%$ & No & $\%$ & No & $\%$ & No & $\%$ & No & $\%$ & No & $\%$ \\
\hline \multirow{2}{*}{ Anti HIV } & Positivo & 7 & 12 & 5 & 9 & 13 & 23 & 15 & 26 & 17 & 30 & 57 & 100 \\
\hline & Negativo & 503 & 12 & 494 & 12 & 886 & 21 & 1130 & 27 & 1213 & 29 & 4226 & 100 \\
\hline \multirow{2}{*}{ Anti Hbs } & Positivo & 35 & 10 & 31 & 9 & 62 & 17 & 82 & 23 & 146 & 41 & 356 & 100 \\
\hline & Negativo & 181 & 11 & 157 & 9 & 319 & 19 & 503 & 30 & 524 & 31 & 1684 & 100 \\
\hline \multirow{2}{*}{ Anti Hcv } & Positivo & 3 & 7 & 2 & 5 & 9 & 22 & 15 & 37 & 12 & 29 & 41 & 100 \\
\hline & Negativo & 254 & 10 & 212 & 8 & 514 & 19 & 816 & 31 & 854 & 32 & 2650 & 100 \\
\hline \multirow{2}{*}{ HbsAg } & Positivo & 3 & 6 & 3 & 6 & 11 & 23 & 17 & 36 & 13 & 28 & 47 & 100 \\
\hline & Negativo & 262 & 10 & 225 & 8 & 535 & 20 & 833 & 31 & 804 & 30 & 2659 & 100 \\
\hline
\end{tabular}

*Fonte: SINAN NET, Bahia, Brasil 2016. 
O teste sorológico evidenciou 57 casos positivos para o vírus HIV. Com relação às condutas tomadas no momento do acidente, observou-se os exames e esquema antirretroviral AZT + 3 TC (Zidovudina e Lamivudina) e em alguns casos acrescidos o Nelfinavir. ${ }^{(14)}$ Os profissionais relatam que a quimioprofilaxia tem diversos efeitos adversos, justificando o abandono do seguimento clinico dos acidentados, que favorece a soroconversão. ${ }^{(4)}$

O vírus da Hepatite $C$ (HCV) só é transmitido de forma eficiente através do sangue e apresentou 41 notificações de laudo positivo. A conduta pós-exposição para esse vírus é através da realização de exames e prosseguir o monitoramento, pois não há profilaxia existente.

O HBsAg é o principal elemento sorológico para diagnostico da hepatite $B$ e esse marcador é o antígeno da superfície do vírus. No período de cinco anos 47 profissionais tiveram o resultado marcando soropositividade que indica a presença no organismo do HBV em atividade, enquanto para negatividade do teste sorológico foram 2.659 casos.

A pesquisa para anticorpo contra antígeno de superfície do vírus da Hepatite B (Anti-HBs) encontrou 356 resultados positivos, que pode corresponder à resposta vacinal anterior, e 1.684 com sorologia negativa, quando o corpo não reproduz o anticorpo vacinal ou o profissional não realizou o esquema da vacina, que esta disponível de forma gratuita na rede pública para os trabalhadores de saúde.

Segundo dados divulgados pelo Centers for Disease Control and Prevention (CDC), os hospitais americanos registram 384.325 casos anualmente de acidentes percutâneos envolvendo trabalhadores da saúde, destacando-se um risco de contaminação de 0,3\% para o vírus da Imunodeficiência Humana (HIV), $6 \%$ a $30 \%$ para o vírus da Hepatite B (HBV) e de $0,5 \%$ a $2 \%$ para Hepatite C (HCV). ${ }^{\left({ }^{15}\right)}$

O processo de soroconversão é variável, depende de alguns fatores, como lesões profundas provocadas por objetos cortantes, presença e volume de sangue envolvido, carga viral, não adesão ao EPI, quando o risco de contaminação existe o profissional tem que ser atendido nas duas primeiras horas e monitorado por no mínimo seis meses com realização de testes sorológicos. ${ }^{(6)}$

\section{CONSIDERAÇÕES FINAIS}

Conclui-se que o perfil é predominantemente com nível técnico (73\%), sexo feminino (78\%), a faixa etária de 30 a 39 anos (36,3\%). O material biológico com maior transmissão é o sangue $(87 \%)$ e a sorologia positiva para HIV apresentam 57 casos.

O período de cinco anos estudados revela que a ocorrência dos acidentes com exposição a material biológico aumentou, entretanto não é possível afirmar se aumentou as notificações ou os acidentes.

A subnotificação decorre por diversos motivos, principalmente o medo em perder o emprego, o sentimento de culpa em relação ao evento e o desconhecimento profissional sobre o preenchimento da CAT (Comunicação de Acidente de TrabaIho), mas também a falta de incentivo da instituição para que as trabalhadoras façam os devidos registros e notificações. ${ }^{(11)}$

O risco ocupacional é diretamente proporcional ao perigo envolvido durante o exercício do trabalho e inversamente proporcional à utilização das medidas preventivas. ${ }^{(13)} \mathrm{O}$ profissional antes de executar algum procedimento, é importante ter o domínio das técnicas, segurança e consciência dos riscos envolvidos.

O código de ética de enfermagem é muito claro no que diz respeito aos danos decorrentes de imperícia, negligência e imprudência em relação à pessoa, família e coletividade. Portanto para a prática segura no trabalho, seguir os protocolos da instituição promove segurança para todos os envolvidos no cuidar.

O desafio de compreender o processo de saúde e doença que envolve o trabalho e trabalhador é dado também aos contratantes de mão-de-obra, quando se deparam com a necessidade de contemplar a subjetividade humana, a inteligência pratica dos colaboradores na construção coletiva da cultura or- 
ganizacional, como forma busca garantir condições de trabalho que favoreçam a satisfação e entusiasmos do profissional, na perspectiva da produção de uma assistência de qualidade, dentro de um ambiente humanizado. ${ }^{(8)}$

A instituição deve desempenhar estudos epidemiológicos, caracterizando os tipos de acidentes e o perfil profissional, para compreender o cenário dos riscos que os trabalhadores estão expostos e nesse contexto intervir com ações em conjuntos de políticas e programas que visam à prevenção.

A Saúde do Trabalhador é uma concepção centrada nos processos de trabalho e no modelo de gestão participativa e corresponsável. As variáveis como: subjetividade, motivação, autonomia, qualidade de vida, gestão de pessoas, cultura organizacional, fornecem bases para elaboração de estratégias entre gestor-profissional, no intuito de desempenhar um trabalho qualificado, produtivo, salubre e que ambos estejam satisfeitos com os resultados.

A implementação de políticas e programas institucionais, que compreenda, profissionais satisfeitos rendem mais e que esse sentimento, amortece as influencias negativas do labor, torna um ambiente mais harmônico, produtivo e saudável.

\section{REFERÊNCIA}

1. Souza FS, Menezes HF, Oliveira MLRR, Paula VG. O trabalhador de enfermagem e os distúrbios osteomusculares relacionados ao trabalho (DORT) - Dialogando sobre a precarização do trabalhador de saúde. Rev Pesq Cuid Fundam. 2011 [Acesso em 2016 abr 18];3(4):2440-2452. Disponível em: http://www. seer.unirio.br/index.php/cuidadofundamental/ article/download/1346/pdf_451

2. Presidência da República (BR). Subchefia para Assuntos Jurídicos. Lei n ${ }^{\circ} 8.231$, de 24 de julho de 1991. Dispõe sobre o seguro de acidentes de trabalho a cargo do INPS e de outras providencias [Internet]. Brasília, DF; 1991. [Acesso em: 2016 mar. O5]. Disponível em: http://www.planalto.gov.br/ccivil_O3/leis/ L8213cons.htm
3. Organização Internacional do Trabalho - Brasil. Promovendo o trabalho decente. [Acesso em: 2016 mar. 15]. Disponível em: http://www.oitbrasil.org.br/content/ apresenta\%C3\%A7\%C3\%A3。

4. Pimenta FR, Ferreira MD, Gir E, Hayashida M, Canini SRMS. Atendimento e seguimento clínico especializado de profissionais de enfermagem acidentados com material biológico. Rev Esc Enferm USP. 2013 [Acesso em: 2016 mar. 8];47(1):198-204. Disponível em: http://dx.doi. org/10.159O/SOO8O-62342013000100025

5. Organização das Nações Unidas - Brasil. OIT: um trabalhador morre a cada 15 segundos por acidentes ou doenças relacionadas ao trabalho. [Acesso em: 2016 mar. 16]. Disponível: http:// www.onu.org.br/oit-um-trabalhador-morre-acada-15-segundos-por-acidentes-ou-doencasrelacionadas-ao-trabalho/

6. Araujo TM, Caetano JA, Barros LM, Lima ACF, Costa RM, Monteiro VA. Acidente de trabalho com exposição a material biológico entre os profissionais de enfermagem. Rev Enf Referência. 2012 [Acesso em: 2016 mar. 30];3(7):7-14 Disponível em: http://dx.doi. org/10.127O7/RIII1182

7. Gallas SR, Fontana RT. Biossegurança e a enfermagem nos cuidados clínicos: contribuições para a saúde do trabalhador. Rev. bras. enferm. 2010 [Acesso em: 2016 mar. 15];63(5):786-792. Disponível em: http://dx.doi. org/10.159O/SO034-71672010000500015

8. Ribeiro AC, Souza JF, Silva JL. A precarização do trabalho no SUS na pespectiva da enfermagem hospitalar. Cogitare Enferm. 2014 [Acesso em: 2016 abr. 14];19(3)569-575. Disponivel em: http://dx.doi.org/10.5380/ ce.v19i3.33034

9. Chiodi MB, Marziale MHP, Mondadori RM, Robazzi MLCC. Acidentes registrados no Centro de Referência em Saúde do Trabalhador de Ribeirão Preto, São Paulo. Rev Gaúch Enferm. 2010 [Acesso em: 2016 mar. O3];31(2):211-217. Disponível em: http://dx.doi. org/10.159O/S1983-14472010000200002

10. Vieira M, Padilha MI, Pinheiro RDC. Analysis of accidents with organic material in health workers. Rev Lat AM Enferm. 2011 [Acesso em: 2016 mar. 10];19(2):332-339. Disponível 
em: http://dx.doi.org/10.1590/SO1O411692011000200015

11. Marziale MHP, Santos HEC, Cenzi CM, Rocha FLR, Trovó MEM. Consequências da exposição ocupacional a material biológico entre trabalhadores de um hospital universitário. Esc Anna Nery Rev Enferm. 2014 [Acesso em: 2016 mar. 12];18(1):11-16. Disponível em: http://www.scielo.br/scielo.php?script=sci_ arttext\&pid=S1414-81452014000100011\&lng= en\&nrm=iso

12. Magagnini MAM, Rocha SA, Ayres JA.O significado do acidente de trabalho com material biológico para os profissionais de enfermagem. Rev Gaúch Enferm. 2011 [Acesso em: 29 mar. 2016];32(2):302-308. Disponível em: http://dx.doi.org/10.1590/S198314472011000200013

13. Lima LM, Oliveira CC, Rodrigues KMR. Exposição ocupacional por material biológico no Hospital Santa Casa de Pelotas - 2004 a 2008. Esc Anna Nery Rev Enferm. 2011
[Acesso em: 2016 mar. O7];15(1):96-102. Disponível em: http://dx.doi.org/10.1590/S141481452011000100014

14. Valim MD, Marziale MHP. Avaliação da exposição ocupacional a material biológico em serviços de saúde. Texto \& Contexto Enferm. 2011[Acesso em: 2016 abr. Ol];2O(spe):138146. Disponível em: http://dx.doi.org/10.1590/ S0104-07072011000500018

15. Centers for Disease Control and Prevention. Healthcare Infection Control Practices Advisory Committee. Guideline for Isolation Precautions: preventing transmission of ifnectious agents in healthcare settings. Atlanta; 2007 [Acesso em: 2016 mar. 31] Disponível: http://www.cdc.gov/ hicpac/pdf/isolation/Isolation2007.pdf 